Hexamethylen and Benzol zu zeigen. Benzin, welches erst mit Permanganatlösung behandelt, nachher längere Zeit mit rauchender Schwefelsäure gestanden und mit Natronlauge gewaschen war, entfärbte das Reagens beim Umschütteln erst nach einigen Minuten; reines Hexamethylen, das wir der Güte des Hru. Professors Zeliusky verdanken, entfärbt schnell, die Flüssigkeit bleibt aber kurze Zeit violet, während beim Schütteln mit Benzol die Farbe augenblicklich versch windet.

Dieser Versuch ist insofern von Interesse, als er zeigt, dass ringförmige, gesättigte Kohlenwasserstoffe unleständiger sind, als die entsprechenden Glieder der Paraffinreihe. Auch gestattet er, die geringsten Spuren von ringförmigen Koblenwasserstoffen im Ligroïn nachzuweisen, wenn man dasselbe vorher durch längeres Schütteln in der Maschine mit Permanganat und Sodalösung ron allen ungesättigten Verbindungen befreit hat.

Sehr merkwürdig ist die grosse Beständigkeit der Bernsteinsäure, welche bei niedriger Temperatur kaum auf das Reagens einwirkt. Geradezu verblüffend wirkt das Experiment, wenn man zu zwei verschiedenen Proben des Reagens einmal Bernsteinsäure und einmal Phtalsäure zugiebt, da Letztere es augenblicklich entfärbt. Die Hoffnung, auf diese Weise das Vorhandensein des Benzolringes nachweisen zu können, wurde indessen nicht erfüllt, da auch die böheren Homologen der Bernsteinsäure, wie z. B. Adipinsäure, das Reagens augenblicklich entfärben. Bemerkenswerth ist noch die ziemlich grosse Bestündigkeit der Oxalsäure.

$\mathrm{Ob}$ das Reagens sich zur Trennung eines Gemisches eignet, muss noch erst durch Versuche ermittelt werden.

405. Frédério Reverdin und Pierre Crépieux: Untersuchungen über den Einfluss der Stellung verschiedener Chromophore im Moleküle auf die Nuance und übrigen Eigenschaften der Farbstoffe.

(Eingegangen am 7. August.)

Wir haben uns die Aufgabe gestellt, zu untersuchen, welcben Einfluss die Stellung der Chromophore $\mathrm{NH}_{2}\left(\operatorname{oder} \mathbf{N}: \mathbf{N}\right.$ ): $\mathrm{NO}_{2}$ und $\mathrm{Cl}$ in Moleküle auf die Nrance, wie auch auf sonstige Eigenschaften der von nitrirten und chlorirten Basen derivirenden Azofarbstoffe ausübe.

Wir begannen diese Studien mit den Farbstoffen, die sich von den Nitro-o-toluidinen - von dieseu sind sämmtliche bekannt - und der Mehrzahl der theoretisch möglicben Monochlortoluidine ableiten 
lassen. Die Farbstoffe, die uns für diese vergleichende Studie dienten, wurden erhalten durch Copulation der obengenannten diazotirten Basen mit 1.4-Naphtolsulfosäure.

Obwohl diese Untersuchungen nicbt zu Resultaten geführt haben, welche uns erlaubt hätten, den Stellungseinfluss der Chromophore in irgend einer allgemeinen Regel auszudrücken, so haben wir immerbin Beobachtungen gemacht, die vielleicht später zur Lōsung dieses Problems beitragen könuteu.

Im Ferneren haben wir für die Darstellung einiger Körper verschiedene Modificationen eingeführt, welche wir an den in Betracht kommenden Stellen anführen werden.

Lassen wir vorerst einige Bemerkungen über die uns als Grundlage dienenden Basen vorangehen.

a) Nitrotoluidine.

Die vier vom Orthotoluidin derivirenden Basen, d. b. die Basen $\mathrm{C}_{6} \mathrm{H}_{3}\left(\mathrm{CH}_{3}\right)\left(\mathrm{NH}_{2}\right)\left(\mathrm{NO}_{2}\right)=1: 2: 3,1: 2: 4,1: 2: 5$ und $1: 2: 6$ siad alle bekannt; und wir möchten nur eine Notiz über die von uns etwas modificirte Darstellung der Derivate $1: 2: 3$ und $1: 2: 5$ hier anbringen.

Nach Lellmann und Würthner') erbält man diese Basen auf folgende Weise:

Man trägt in $31 / 2$ Theile eines Gemisches von 3 Theilen rauchender Salpetersäure und 1 Theil Eisessig, welches sich in einem tnit Eis gekühlten Kölbchen befindet, 1 Theil Orthoacettoluid in kleinen Portionen ein, und zwar so, dass die Temperatur $7-8^{0}$ nicht übersteigt. Hierauf lässt man 12 Stdn. bei Zimmertemperatur stehen und giesst dann in wenig Wasser, filtrirt und wäscht aus. Statt der Verseifung mit alkoholischer Kalilauge, durch welche nur das Acetylderivat der Base $1: 2: 5$ angegriffen würde, und nachheriger mechanischer Trennung (Lellmann und $W$ ürthner) der Krystalle der Base $1: 2: 5$ von denjenigen des Acetylproductes der Base $1: 2: 3$, welch' Letztere dann mit Salzsäure zu verseifen wären, haten wir es vorgezogen, folgendermaassen zu arbeiten:

Das robe Nitrirungsproduct wird während 2 Stunden mit 3 Theilen Salzsäure erbitzt, wodurch beide Derivate verseift wurden. Destillirt man hierauf die salzsäurebaltige Lösung dieser Miscbung mit Wasserdampf, so wird das salzsaure Salz des Nitrotoluidins $1: 2: 3$ dissociirt, und die freie Base destillirt mit den Wasserdämpfen über, wäbrend das Chlorhydrat der Base 1:2:5 im Kolben zurückbleibt. Diese Letztere wird gewonnen, indem man mit Natronlauge behandelt, filtrirt, auswäscht und die Base alsdanı aus Alkohol umkrystallisirt.

1) Ann. d. Chem. 228, 240. 
Wir erhielten nach dieser Methode aus $15 \mathrm{~g}$ Acettoluid $12 \mathrm{~g}$ des Nitroproductes, aus welchem wir nach dem Verseifen gewannen:

$$
\begin{aligned}
& 5.3 \mathrm{~g} \text { Nitrotoluidin } 1: 2: 3, \\
& 3.4 \mathrm{~g}, 1: 2: 5 .
\end{aligned}
$$

Für die Darstellung des 1:2:3-Nitrotoluidins kennt man noch eine andere Methnde, diejenige yon Gnehm und Blumer ${ }^{1}$ ).

Nach derselben wird das rorher sulfurirte Ortboacettoluid nitrirt, die Sulfo Gruppe alsdann durch Erhitzen eliminirt und die Base $1: 2: 3$ mit Wasserdampf überdestillirt.

Die $\Lambda$ usbente bei diesem Verfahren übersteigt nicht diejenige, welche man nach der vou uns verbesserten Methode ron Lellmann und Würthuer erhalten kann; zudem bildet sich nur die Base 1:2:3, denn der Zweck der Gnehm-Blumer'schen Methode war gerade, die Bildung der Base $1: 2: 5$ zu verhindern.

\section{b) Chlor-toluidine.}

1. Derivate des $o$-Toluidins,

$\mathrm{C}_{6:} \mathrm{H}_{3}\left(\mathrm{CH}_{3}\right)\left(\mathrm{NH}_{2}\right)(\mathrm{Cl})=1: 2: 3,1: 2: 4,1: 2: 5$ und $1: 2: 6$.

Das Derivat $1: 2: 3$ haben wir bis jetzt noch nicht erhalten köpnen.

Die drei anderen Basen sind schon bekannt.

Das o-Chlortoluidin 1:2:5 wurde von Lellmann und $\mathrm{K}\left(\mathrm{trz}^{2}\right)$ dargestellt, indem sie das Orthoacettoluid in essigsaurer Lösung mit Chlorgas cblorirten. Wir fanden es vortheilhafter, mit Natriumchlorat und Salzsäure zu chloriren.

Beispiel. Wir lösten $15 \mathrm{~g} o$-Acettoluid in $50 \mathrm{ccm}$ Eisessig und setzten $24 \mathrm{~g}$ Salzsäure hinzu. In das abgekühlte Gemisch liessen wir tropfenweise eine Lösung voll $6.6 \mathrm{~g}$ Natriumchlorat in $30 \mathrm{ccm}$ Wasser einfliessen, und zwar so, dass die Temperatur $20^{\prime}$ nicht überstieg. Nachdem alles Chlorat eingetragen war, worde eine Stunde bei gewöbnlicher Temperatur stehen gelassen, hierauf mit Wasser gefällt, filtrirt und aus Alkohol umkrystallisirt. Das einmal umkrystallisirte Product (Schmp. 1350 statt 140 ${ }^{n}$ ) wurde verseift mittels einer concentrirten alkoholischen Kalilauge - als Verseifungsmittel kann auch Salzeäure angewendet werden - und mit Wasserdampf überdestillirt.

Die Base 1:2:6 würde vom entsprechenden Nitrotoluidin ausgehend dargestellt. Es wurde so zuerst das correspondirende Nitrochlortoluol erhalten (nach der Methode ron Sandmeyer), welches alsdann reducirt wurde.

Wir beobachteten bei Gelegenheit dieser Transformationen, dass sich die Diazotirung der Nitrobase langsam und unvollständig vollzieht,

i) Ann. d. Chem. 304, 105.

2) Ann. d. Chem. 231, 317. 
und dass sich alsdann bei Ausführung der Sandmeyer'schen Reaction, ausser dem gewünschten Chlornitrotoluol, noch ein zweites Product bildet, das mit Wasserdämpfen nicht flüchtig ist. Diese zweite, braungefärbte, pulverige Substanz schmolz, aus Benzol und Ligroïn umkrystallisirt, bei $198^{\circ}$.

2. Derivate des $m$-Toluidins,

$\mathrm{C}_{6} \mathrm{H}_{3}\left(\mathrm{CH}_{3}\right)\left(\mathrm{NH}_{2}\right)(\mathrm{Cl})=1: 3: 2,1: 3: 4,1: 3: 5$ und $1: 3: 6$.

Von diesen Verbindungen sind alle bekannt.

Bei der Darstellung der Base 1:3:2 mit Hülfe des Nitrotoluidins $\mathrm{C}_{6} \mathrm{H}_{3}\left(\mathrm{CH}_{3}\right)\left(\mathrm{NH}_{2}\right)\left(\mathrm{NO}_{2}\right)=1: 2: 3$, beobacbteten wir ebenfalls bei der Ausführung der Sandmeyer'schen Reaction die Bildung eines zweiten Productes in ausehnlicher Quantität (ungefähr $50 \mathrm{pCt}$.). Dasselbe war mit Wasserdämpfen nicht füchtig, schmolz bei $106^{\circ}$ und krystallisirte in gelben Nadeln.

Bei der Darstellung der Base 1:3:4 constatirten wir die Bildung eines analogen Productes, das in gelblich-braunep Nadeln krystallisirte und bei $141^{\circ}$ schmolz.

Als Nebenproduct bei der Darstellung der Base $1: 3: 6^{1}$ ) erhielten wir eine rothe, krystallisirende Substanz.

Diese secundären Producte, welche unseres Wissens noch nicht bescbrieben worden sind, werden wir später näher untersuchen.

$$
\begin{aligned}
& \text { 3. Derivate des } p \text {-Toluidins, } \\
& \mathrm{C}_{6} \mathrm{H}_{3}\left(\mathrm{CH}_{3}\right)\left(\mathrm{NH}_{2}\right)(\mathrm{Cl})=1: 4: 2 \text { und } 1: 4: 3 \text {. }
\end{aligned}
$$

Beide Basen sind bekannt.

Die Base 1:4:3 wurde dargestellt sowobl durch Chloriren und nachträgliches Verseifen des $p$-Acettoluids nach den von uns modificirten Methoden von Wróblewsky ${ }^{2}$ ) und von Lellmann und $\left.\mathrm{K} / \mathrm{tg}^{3}\right)$, wie auch durch Reduction des $m$-Chlor-p-Nitrotoluols. Die Darstellung des letzteren Körpers werden wir in einer anderen Abhandluug darlegen.

Bebufs Chlorirung des $p$-Acettoluids geben wir 35 g Salzsäure zu einer Lösung von $22.5 \mathrm{~g} p$-Acettoluid in $75 \mathrm{ccm}$ Eisessig; alsdann lassen wir zu dem geküblten Gemische eine Lösung von $10 \mathrm{~g}$ Natriumchlorat in $45 \mathrm{ccm}$ Wasser tropfenweise zufliessen. Nach 12-stündigem Stehenlassen bei gewöhnlicher Temperatur wird alsdann mit Wasser gefällt, filtrirt, aus verdünntem Alkohol (5 Th. Wasser auf 1 Th. Alkohol) umkrystallisirt und mit Salzsäure rerseift.

1) Dis Base 1:3:6 wurde dargestellt durch Chlorirung des m-Acettoluidins. Siehe nächste A błaodlung.

2) Ann. d. Chem. 168, 196.

3) Ann. d. Chem. 231, 311. 
Prüfung der von den Nitro- und Chlor-Toluidinen derivirenden Farbstoffe.

Zur Erleichterung der Uebersicht fübren wir die ConstitutionsFormeln der Basen, deren Farbstoffe untersucht worden waren, hier unten an und bezeichnen im Verlaufe dieser Bescbreibung die in Frage stehenden Verbindungen mit den ihnen zukommenden Nummern.

\section{Nitrotoluidine.}

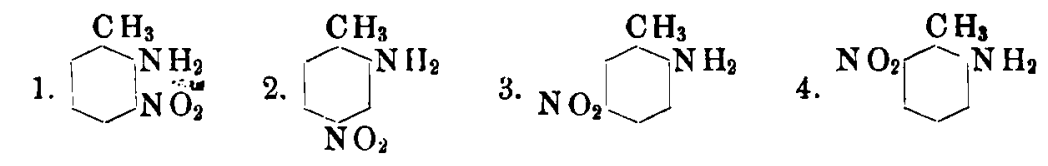

Chlortoluidine.

5 .<smiles>NC1CCCCC1</smiles>

9.<smiles>CC1CCCCC1</smiles>

6.<smiles>C[NH2+]C1CCCCC1</smiles>

10.<smiles>CC1CCNCC1</smiles>

7.<smiles>CC1CCCCC1</smiles>

11.<smiles>CC1CCCCC1</smiles>

8.<smiles>CC1CCCNC1</smiles>

12.<smiles>Cl[AlH]1CCCCC1</smiles>

Farbstoffe, erhalten ans den obengenannten diazotirten Basen durch Copulation init 1.4-Naphtolsulfosäure.

Die Ausfärbungen dieser Farbstoffe wurden auf gewöhnlicher Wolle in schwefelsaurem Bade unter Zusatz von Glaubersalz ausgeführt.
a) Farbstoffe aus den Nitrotoluidinen.

Diese Farbstoffe geben orangerothe bis rothe Ausfärbungen, und 2war sind die Nuancen der Base 1, 2 und 4 fast identisch (rothorange), während der von der Base 3 sich alleitende Farbstoff bedeutend röther ist.

Was die Solidität dieser Farbstoffe gegenüber dem Lichte anbetrifft, so sind charakteristische Differenzen zu constatiren. So ist der Farbstoff der Base 1 sehr wenig lichtecht, derjenige der Base 2 zeigt dagegen eine bedeutend grössere Echtbeit, derjenige der Base 4 ist im Vergleich zu dem vorhergehenden in die zweite und derjenige der Base 3 in die dritte Stelle zu setzen. Aus diesen Thatsachen ist zu entnehmen, dass der Farbstoff, in welchem die Gruppe $. N: N$. sich in unmittelbarer Nähe der Gruppe $\mathrm{NO}_{2}$ befindet, am wenigsten lichtecht ist. Wir haben im Weiteren beobachtet, dass der dem Nitrotoluidin $\left(\mathrm{CH}_{3}\right)\left(\mathrm{NH}_{2}\right)\left(\mathrm{NO}_{2}\right)=1: 4: 3$ entsprechende Farbstoff, in welchem sich also die Gruppe $. N: N$. ebenfalls in directer $N a ̈ h e$ 
der Gruppe $\mathrm{NO}_{2}$ befindet, ebenfalls - obschon etwas mehr - wenig lichtecht ist.

In dem Derivat der Base 1 sind alle 3 Gruppen benachbart; vielleicht muss man diesen Unstande die geringe Lichtechtheit znschreiben.

Was die übrigen Eigenschaften obiger Farbstoffe anbelangt, $\mathbf{s} 0$ ist $\mathbf{z u}$ bemerken, dass derjenige der Base 1 sehr leicht, derjenige der Base 3 sehr wenig löslich ist. Der Letztere unterscheidet sich auch noch durch folgendes Verhalten von den anderen: In Wasser suspendirt, geht er auf Zusatz von Soda oder Natronlauge (bei Zusatz von Soda erst beim Erhitzen) mit tiefvioletrotber Farbe in Lösung.

\section{b) Farbstoffe aus den Chlortoluidinen.}

Die Farbstoffe der den Nitro- $\omega$-Toluidinen entsprechenden Chloro-Toluidine geben eine bedeutend röthere und lebhaftere Nuance.

Vergleicht man die von den Nitrotoluidinen $\mathrm{C}_{6} \mathrm{H}_{3}\left(\mathrm{CH}_{3}\right)\left(\mathrm{NH}_{2}\right)\left(\mathrm{NO}_{2}\right)$ $=1: 4: 3$ sich ableitenden Farbstoffe mit den aus den entsprechenden ChInrtoluidiuen dargesteliten, so findet man, dass die Letzteren in Gegentheil eine gelblichere Nuance besitzen.

Beim Vergleich der Nuancen der aus den verschiedenen Chlortoluidinen erhaltenen Farbstoffe haben wir Folgendes beobachtet: Alle diese Farbstoffe besitzen eine Nuance zwischen roth und orange, mehr oder weniger stark in das Rothe ziehend. Diejeniges der Basen 6 und 5 sind bedentend röther als die anderen; diejenigen der Basen $9,10,12,8$ und 11 sind unter sich fast gleich, aber von gelblicherer Nuance als 6 und 5; die Base 7 endlich giebt den gelblichsten Farbstoff von allen.

Die Derivate des o.Toluidins, mit Ausuahme desjenigen, in welchem das Chlor sich in Ortho gegenüber der Methylgruppe befiudet, unterscheiden sich sehr deutlich durch eine röthere Nuance; diejenigen des $m$-Toluidins und des $p$-Toluidins sind alle gelblicher.

Was die Solidität obiger Farbstoffe gegenüber dem Lichte anbelangt, so haben wir analoge Beobachtungen gemacht, wie wir sie bei den Derivaten der Nitrotoluidine constatirten. Der Farbstoff der Base 8 ist gegenüber dem Lichte der an wenigsten echte; hierauf folgen diejenigen der Basen 9 und 12, welche ebenfalls nicht sehr lichtecht sind, und schliesslich derjenige der Base 7; diejenigen der anderen Basen dagegen zeigen eine sehr gute Lichtechtheit. Die Lichtunechtheit scheint also ron der directen Nachbarschaft des Chlors mit der Amidogruppe herzurühren und ist noch besonders ausgesprochen, wens alle drei Gruppen, $\left(\mathrm{CH}_{3}\right)(\mathrm{Cl})\left(\mathrm{NH}_{2}\right)$, in Ortho stehen und zugleich Chlor mit Amido direct benachbart ist (bei Base 8).

Was die Löslichkeit anbetrifft, so sind die Farbstoffe der Basen 5,10 und 11 die löslichsten; die anderen sind weniger leicht löslich, 
und besonders wenig löslich in der Kälte sind diejenigen der Basen 8 und 9. Das Derivat der Base 6 verhält sich, was die Löslichkeit anbelangt, wie sein correspondirendes Nitroderivat.

Dies wären die Beobachtungen, welche wir bei den vergleichenden Studien der in Frage kommenden Farbstoffe Gelegenheit hatten zu machen.

Wir wollen nicht schliessen, ohne Hrn. Dr. Keller, welcher uns bei dieser Arbeit geholfen hat, wie auch den Höchster Farbwerken, welche in zuvorkommender Weise die Mehrzahl der nöthigeu Producte lieferten und die Ausfärbungen besorgten, unseren Dank auszusprechen.

406. Frédéric Reverdin und Pierre Crépieux: Ueber die Chlorirung des m-Acettoluids.

(Eingegangen am 7. August.)

Durch Chlorirung des $m$-Acettoluids nittels Natriumchlorat und Salzsäure erhielten wir, je nach den angewandten Verhältnissen der Chlorirungsmittel, ein Mono-, ein Di- oder ein Tri-Chlorderivat.

Das Monochlor-m-acettoluid, welches, wie weiter unten zu erseben ist, der Formel $\mathrm{C}_{6} \mathrm{H}_{3}\left(\mathrm{CH}_{3}\right)(\mathrm{Cl})\left(\mathrm{NH} . \mathrm{C}_{2} \mathrm{H}_{3} \mathrm{O}\right)(1: 2: 5)$ entspricht, wird auf folgende Weise erhalten:

Man löst $18 \mathrm{~g} \mathrm{~m}$-Acettoluid in $60 \mathrm{ccm}$ Eisessig. Zu dieser Lösung fügt mau in kleinen Portionen $17 \mathrm{ccm}$ reine Salzsüure; dann setzt man zu der kalt gewordenen Mischung eine Lösung vou $5.2 \mathrm{~g}$ Natriumchlorat in $20 \mathrm{ccm}$ Wasser und zwar so, dass die Temperatur $20^{\circ}$ nicht übersteigt. Das Ganze lässt man nun 15-20 Stdn. bei Zimmertemperatur stehen und versetzt alsdann das Reactionsproduct mit Wasser. Hierbei scheidet sich das Monochlor-m-acettoluid als ein braunes Oel ab; dieses wird vom $W$ asser getrennt und dann in Aether aufrenommen; nach dem Verdunsten des Aethers wird der Rückstand direct verseift, durch Erhitzen mit Salzsäure auf dem Wasserbade.

Das Product löst sich rasch und setzt sich dann als Chlorbydrat der Base krystallinisch ab. Die Lösung des Chlorhydrats wird durch Zusatz von Natronlauge schwach alkalisch gemacht und dann destillirt. Man erhält auf diese Weise eine feste Base, welche, aus Ligroïn umkrystallisirt, bei 83" schmilat und deren A ce tylderivat einen Schmelzpunkt von $89^{\circ}$ zeigt. Die Eigeuschaften der erbaltenen Base stimmen vollkommen mit denen des $o$-Cblor-m-toluidins überein, wie sie zuerst von Wróblewski ${ }^{1}$ ), dann ron Goldschmidt und $\mathrm{Höuig}{ }^{2}$ )

1) Ann. d. Chem. 168, 206.

3) Diese Berichte 19, 2443; 20, 200. 